\title{
On Novel Efficient Wireless Access Network Design Heuristic Algorithms For QoS Multiservice Networks
}

\author{
Vasilios Pasias \\ ONEX COMPANY S.A. \\ 87, Kon.Palaiologou Str., \\ Chalandri , 1523, Athens, Hellas \\ Fax: +302104310875
}

\author{
Dimitrios A. Karras \\ Sterea Hellas Institute of \\ Technology, Dept. Automation, \\ Chalkis, P.C 34400, Greece, \\ e-mails: dakarras@teiste.gr, \\ dimitrios.karras@gmail.com, \\ Dimitrios.karras@ieee.org
}

\author{
Rallis C. Papademetriou \\ School of Engineering, University \\ of Portsmouth, UK \\ e_mail: \\ rallis.papademetriou@port.ac.uk
}

\begin{abstract}
This paper presents two novel heuristic algorithms for the design of wireless access networks (QoS-HWNDA-1, QoSHWNDA-2) adapted in the case of QoS networks, which is the prevalent situation in the evolution of 5G technology. Emphasis was given to the design of CDMA based wireless networks and Fixed Wireless networks considering QoS architectures. The objectives of these methods are first to place a number of access points/base stations in a number of candidate sites and then to assign a number of fixed wireless terminals to the selected access points/base stations, while ensuring QoS requirements. Both methods are based on Graph Theory and they are essentially greedy algorithms. Except from capacity constraints, wireless reception characteristics are also considered. The algorithms are capable of designing medium and large-scale networks at polynomial time. Both algorithms were compared with a rival optimization problem through a series of tests. The results indicate that as regards design costs the performance of the proposed heuristic algorithms is very close to this of the equivalent optimization problem. The solution times for the proposed heuristic algorithms are smaller especially when the number of the candidate access points/base stations becomes large.
\end{abstract}

Keywords- Wireless Access Networks, QoS Networks, Multiservice Networks, Integer Linear Programming (ILP), Heuristic Algorithms, Graph Theory

\section{INTRODUCTION}

Recent years have witnessed tremendous developments as regards wireless network access. New technologies enable full wireless connectivity to a wide range of devices (e.g. PCs, smartphones, Laptops, PDAs, tablets etc.) allowing them to use all kinds of services (e.g. voice, video, data, conferencing, social networking) [1] without the need for wired communications. However, in this paper emphasis is given to methods for the design of in-door Coded Division Multiple Access (CDMA) based wireless networks and Fixed Wireless (e.g. Wireless Local Loop - WLL) networks [2].. The aforementioned networks are referred to as wireless access networks. In these networks, two main types of network components are considered: the access point/base station and the fixed wireless user/terminal. The fixed wireless terminals (test points) claim for access to the network and the access points/base stations connect the terminals to the network. In fact, consider the set $\mathrm{T}$ of fixed wireless terminals and the set $\mathrm{S}$ of possible (predefined) locations for the placement of access points/base stations. Each terminal $i \in \mathrm{T}$ should be connected to only one access point/base station $\mathrm{j} \in \mathrm{S}$. Each access point/base station together with its associated terminals, constitute an access subnetwork. Moreover, the access points/base stations can be connected to one or more other subnetworks (e.g. the backbone subnetwork) [2]. A typical network that includes wireless access subnetworks is illustrated in Figure 1. Note that the term base station will be used instead of the term access point from now on.

The process of wireless network planning basically consists of two phases:

1. Base station placement. A number of base stations must be placed in a number of (predefined) candidate sites, essentially one base station in each selected site.

2. Wireless terminal (test point) allocation to the selected base station(s) in phase 1 .

Practically, both phases can be considered as one. However, the design cost that here refers to base station placement (installation) and operation, should be minimum. The design process takes into consideration two basic factors:

A. Base station capacity. The number of terminals assigned to each base station $\mathrm{j} \in \mathrm{S}$ is restricted by its total terminal throughput (switching speed) or user/port capacity $\mathrm{W}_{\mathrm{j}}$. See Section II for more details on base station capacity. 
B. Received power $\mathrm{P}_{\mathrm{ij}}$ at fixed wireless terminal $\mathrm{i} \in \mathrm{T}$ that is emitted from base station $\mathrm{j} \in \mathrm{S}$ only. The received power at terminal $\mathrm{i}$ emitted from base station $\mathrm{j}$ only, basically depends on the transmitted power by the base station, the gain of each base station or terminal antenna, the actual height of each base station or terminal antenna, the operating frequency, the distance between the base station and the terminal and also the environmental and terrain conditions [1-5]. For the present problem, provided that all other conditions are met (e.g. there is enough throughput or user/port capacity at base station $\mathrm{j}$ ), terminal $\mathrm{i}$ can only be allocated to base station $\mathrm{j}$ when $\mathrm{P}_{\mathrm{ij}}$ is greater than or equal to a specified reception threshold $\mathrm{REC}_{\mathrm{i}}$.

Unfortunately, today there are no systematic methods to address the problem of wireless network planning, especially regarding CDMA based networks, fixed wireless and UMTS access networks. In fact, the planning process is mainly based on empirical approaches [2]. Nevertheless, recent design approaches [6, 7], based on Integer Linear Programming (ILP) and Mixed Integer Programming (MIP) techniques, try to address the above problem in a rather interesting way that it may be very advantageous in practice. In [6] a wireless system planning procedure, especially suited for in-door CDMA networks, is presented which is based on a complex ILP model. However, in [6] no simulation results regarding topologies with more than 27 wireless users are provided. In [7] a series of complex MIP optimization models especially suited for UMTS

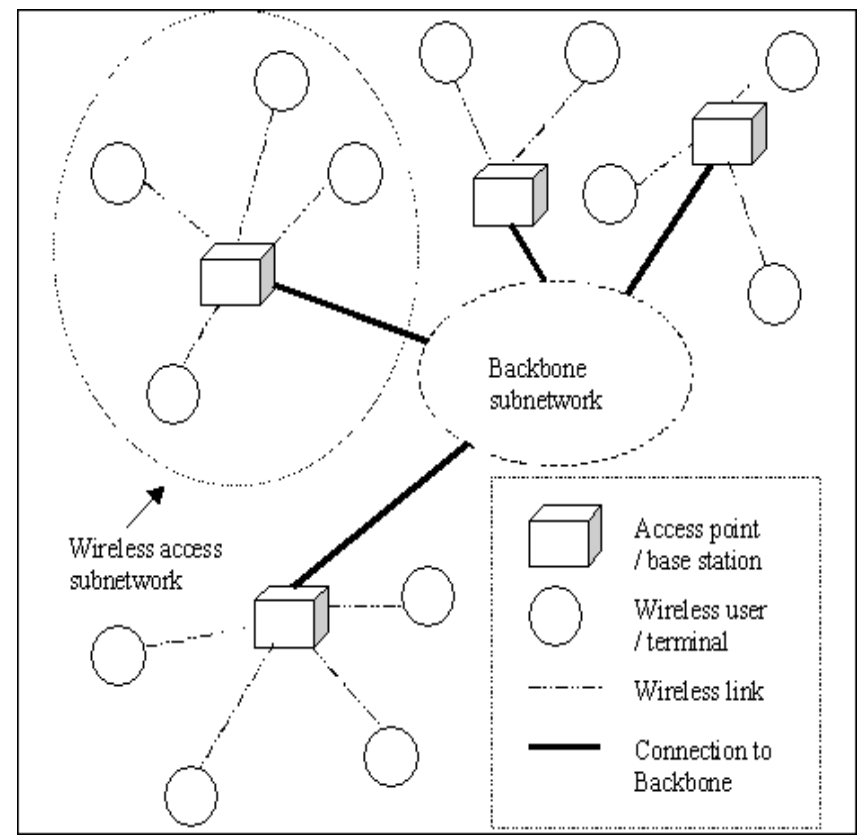

Figure 1. Typical wireless network infrastructure example

base station planning are presented. Note that in Section II an optimization ILP model, essentially based on the work included in $[6,7]$, is analytically described. The aforementioned optimization methods require a lot of time for the solution of the design problems they involve, especially when the number of the network devices increases. In fact, these methods, especially the method described in [7], cannot solve large network design problems in Polynomial or NonPolynomial (NP) time [7]. For the solution of such problems in Polynomial or NP time, near-optimal heuristic algorithms are thus required. In [7] heuristic methods for UMTS base station planning, involving greedy randomized procedures and Tabu Search algorithms are presented that provide efficient solutions in Polynomial time.

In this paper two new heuristic algorithms namely the Heuristic Wireless Network Design Algorithm 1 (QoSHWNDA-1) and the Heuristic Wireless Network Design Algorithm 2 (QoS-HWNDA-2) are presented, adapting the older WNDA-1 and WNDA-2 proposed by the same authors [14]. These algorithms can be used for the design of small $(<$ 20 network devices), medium ( 50 devices) and large $(>100$ devices) wireless access networks, such as CDMA based and fixed wireless (WLL) networks. Specifically, the objectives that motivated the development of the new heuristic design methods are:

- The design methods should be capable of designing efficient medium and large-scale access networks at Polynomial time.

- Their performance should be similar to that of the optimization methods described in Section II and also in $[6,7]$.

The QoS-HWNDA-1 and QoS-HWNDA-2 methods are thoroughly described in Sections III and IV respectively. In Section V, simulation results regarding the comparison between a slightly modified version of the standard base station placement and terminal assignment optimization ILP problem described in Section II and the heuristic algorithms QoSHWNDA-1 and QoS-HWNDA-2 are presented. This comparison provides an efficient measure in order to validate the performance of the new developed heuristic methods. Note that in the simulation tests, small, medium and large network topologies were considered. Finally, Section VI concludes the paper.

\section{The Standard Base Station Placement \& Terminal ASSIGNMENT ILP PROBLEM ADAPTED TO QOS MULTISERVICE NETWORKS}

The formulation of this ILP problem is based on [6, 7]. Note that the algorithm that solves an ILP problem is deterministic and has the advantage of always delivering the optimal solution if there is one $[6,7]$.

Consider $|\mathrm{T}|$ terminals and $|\mathrm{S}|$ base stations. The cost of connecting the terminal $i \in T$ to the base station $j \in S$ is $c_{i j}$. $c_{i j}$ is proportional to the length between the terminal $i$ and the base station $\mathrm{j}$. The cost of placing and/or using the base station $\mathrm{j}$ is $\mathrm{Cj}$. The capacity of the base station $\mathrm{j}$ is $\mathrm{W}_{\mathrm{j}}$. Terminal $\mathrm{i}$ requires $\mathrm{w}_{\mathrm{ij}}$ units of capacity at base station $\mathrm{j}$. Note that if base station capacity refers to user (port) capacity on the base station, then $\mathrm{w}_{\mathrm{ij}}=1$ and $\mathrm{W}_{\mathrm{j}}$ is the maximum allowable number of users that can be associated with the base station $\mathrm{j}$; 
otherwise $\mathrm{w}_{\mathrm{ij}}$ refers to terminal throughput and $\mathrm{W}_{\mathrm{j}}$ refers to the total amount of terminal workload that the base station $\mathrm{j}$ can handle (switching speed). $\mathrm{P}_{\mathrm{ij}}$ is the received power at terminal $\mathrm{i}$, which is emitted from base station $\mathrm{j}$ only and $\mathrm{REC}_{\mathrm{i}}$ is the power reception bound for terminal $i$. The decision variables for the problem are:

- $\mathrm{X}_{\mathrm{ij}}$ : Connection establishment integer variable between base station $\mathrm{j} \in \mathrm{S}$ and wireless terminal $\mathrm{i} \in \mathrm{T}$; it is $\mathrm{X}_{\mathrm{ij}}=1$ if a connection is established, $\mathrm{X}_{\mathrm{ij}}=0$ otherwise.

- $\mathrm{Y}_{\mathrm{j}}$ : Base station placement integer variable; it is $\mathrm{Y}_{\mathrm{j}}=1$ if a base station is placed at the potential transmission site $\mathrm{j}$ $\in \mathrm{S}, \mathrm{Y}_{\mathrm{j}}=0$ otherwise.

The objective function of the problem tries to minimize the number of the required base stations, the base station placement/utilization cost and the cost of connecting terminals to base stations, as well as to maximize the received signal strength over all reception sites.

$$
\begin{aligned}
& \min Z=\left(\sum_{j=1}^{S} C_{j} \bullet Y_{j}\right)+\left(\sum_{i=1}^{T} \sum_{j=1}^{S} c_{i j} \bullet x_{i j}\right) \\
& -\left(\sum_{i=1}^{T} \sum_{j=1}^{S} P_{i j} \bullet x_{i j}\right)
\end{aligned}
$$

The following constraint guarantees that each terminal is associated with one base station only

$$
\sum_{j=1}^{S} x_{i j}=1, \forall i \in T
$$

The next constraint ensures that the received power Pij at terminal $\mathrm{i}$ is equal to or greater than the power reception bound $\mathrm{REC}_{\mathrm{i}}$

$$
\sum_{j=1}^{S} P_{i j} \bullet x_{i j} \geq R E C_{i}, \forall i \in T
$$

Generally, the received power $\mathrm{Pij}(\mathrm{dBW})$ at terminal $\mathrm{i}$ is given by the following relation:

$P_{i j}=P_{j i}+G_{i}+G_{j}-20 \bullet \log (4 \bullet \pi \bullet d \bullet f / c)-L_{\text {tot }}$

where Pji: transmitted power $(\mathrm{dBW})$ by the base station $\mathrm{j}$ (in the direction of terminal i), Gi: terminal i antenna gain (dBi), $\mathrm{Gj}$ : base station $\mathrm{j}$ antenna gain $(\mathrm{dBi}), \mathrm{d}$ : distance between the base station $j$ and terminal $i(m)$, f: operating frequency $(\mathrm{kHz})$, c: speed of light $(300000 \mathrm{~km} / \mathrm{sec})$ and Ltot: total power losses (dB) due to environmental (atmospheric) and terrain conditions. Practically, empirical wireless propagation models are used in order to model terrestrial radio transmission, such as the next:
- Ibrahim \& Parsons Model [2].

- Two-Ray Ground Reflection Model [2, 8].

- CCIR Recommendation 370-4 Model [9].

- Basic CCIR Model [10].

- Hata Model [11].

- Walfish-Ikegami Line-Off-Sight Model [12].

- Walfish-Ikegami non-Line-Off-Sight Model [12].

For example, according to the Ibrahim \& Parsons Model for open areas [2], the received power Pij $(\mathrm{dBW})$ at terminal $\mathrm{i}$ is given by the formula below:

$$
\begin{aligned}
& \quad P_{i j}=P_{j i}+G_{i}+G_{j}-20 \bullet \log \left(d^{2} / h_{i} \bullet h_{j}\right)-20-f / 40- \\
& -0.18 \cdot L+0.34 \cdot H
\end{aligned}
$$

where hi: terminal i antenna height $(\mathrm{m}), \mathrm{hj}$ : base station $\mathrm{j}$ antenna height $(\mathrm{m})$, L: land usage factor (\%), f: operating frequency in $\mathrm{MHz}$ and $\mathrm{H}$ : height difference (in meters) between the $0.5 \mathrm{~km}$ squares containing terminal $\mathrm{i}$ and base station j.

The following constraint guarantees that the capacity bounds of the base stations are not violated.

$$
\sum_{i=1}^{T} w_{i j} \bullet x_{i j} \leq W_{j} \bullet Y_{j}, \forall j \in S
$$

In the case of QoS networks it is important to ensure that the utilization of the total network capacity is maximized, something not inherent in the previous formalism.

Therefore, we herein propose a modification of the equation (1) above as follows

$$
\begin{aligned}
& \min Z=\left(\sum_{j=1}^{S} C_{j} \bullet Y_{j}\right)+\left(\sum_{i=1}^{T} \sum_{j=1}^{S} c_{i j} \bullet x_{i j}\right) \\
& -\left(\sum_{i=1}^{T} \sum_{j=1}^{S} P_{i j} \bullet x_{i j}\right)+ \\
& +\sum^{S} \sum_{i=1}^{T}\left(w_{i j} \bullet x_{i j}-W_{j} \bullet Y_{j}\right)^{2}, \forall i \in T, j \in S
\end{aligned}
$$

where, obviously, the last term of the above equation (1a) attempts to minimize capacity or bandwidth not utilized, thus, maximizing network utilization.

However, if the solution of the problem is infeasible then an LP-relaxed version of it is solved. Nonetheless, only integer (0 or 1$)$ results are finally considered. 
III. HEURISTIC WIRELESS NETWORK DESIGN ALGORITHM1 (QOS-HWNDA-1)

The Heuristic Wireless Network Design Algorithm-1 (QoS-HWNDA-1), which is based on the Add algorithm [13], is described below.

1) All wireless terminals $i \in T$ are connected directly to a predefined center with infinite capacity. The connection costs to the center are infinite.

2) A base station $\mathrm{j} \in \mathrm{S}$ is added at each site and the cost savings as well as the increment of network communication capacity utilization, obtained by this addition, are evaluated.

3) After trying a number of base station placements, the algorithm selects the most cost saving base station but, based on equation (1a). on the other hand, maximizing network communication capacity utilization.

4) The most cost efficient wireless terminals are associated with it provided that the base station capacity $\mathrm{W}_{\mathrm{j}}$ is not exceeded and that the received power $\mathrm{P}_{\mathrm{ij}}$ by the terminal $\mathrm{i}$ $\in \mathrm{T}$ is greater than or equal to the predefined reception threshold $\mathrm{REC}_{\mathrm{i}}$. For information regarding the calculation of the received power $\mathrm{P}_{\mathrm{ij}}$, see Sections I and II.

5) After each base station is selected, the savings by adding an additional base station change and all potential savings and utilization improvements are re-evaluated by examining separately each of the terminals and by examining again network total capacity utilization maximization with respect to the criterion above

$\min Z_{0}=$

$\sum^{S} \sum_{i=1}^{T}\left(w_{i j} \bullet x_{i j}-W_{j} \bullet Y_{j}\right)^{2}, \forall i \in T, j \in S$

6) The algorithm stops if no more cost saving base stations can be found that satisfies maximization of network communication capacity utilization. Finally, all terminals will be connected to base station, provided that there is enough capacity to the base stations.

\section{HEURISTIC WIRELESS NETWORK DESIGN ALGORITHM-2 (QOS-HWNDA-2),}

The Heuristic Wireless Network Design Algorithm-2 (QoSHWNDA-2), based on the Drop algorithm [13], is demonstrated next.

1) The following Terminal Assignment (TA) ILP optimization problem is solved:

Consider $|\mathrm{T}|$ terminals and $|\mathrm{S}|$ base stations. The cost of connecting terminal $\mathrm{i}$ to base station $\mathrm{j}$ is $\mathrm{c}_{\mathrm{ij}}$ (proportional to the length between the terminal $i$ and the base station $j$ ). The capacity of the base station $\mathrm{j}$ is $\mathrm{W}_{\mathrm{j}}$. Terminal $\mathrm{i}$ requires $w_{i j}$ units of capacity at base station $j$. The decision (integer) variable is: $x_{i j}$, that is $\mathrm{x}_{\mathrm{ij}}=1$ if terminal $\mathrm{i}$ is assigned to the base station $\mathrm{j}$; otherwise $\mathrm{x}_{\mathrm{ij}}=0$.

The next objective function tries to minimize the cost $\mathrm{Z}$ of connecting the terminals to base stations.

$$
\min \quad Z=\sum_{i=1}^{T} \sum_{j=1}^{S} c_{i j} \bullet x_{i j}
$$

The following constraint guarantees that each terminal is associated with a base station.

$$
\sum_{j=1}^{S} x_{i j}=1, \forall i \in T
$$

The next constraint ensures that the received power $\mathrm{P}_{\mathrm{ij}}$ at terminal $\mathrm{i}$, emitted from base station $\mathrm{j}$ only, is equal to or greater than the power reception bound $\mathrm{REC}_{\mathrm{i}}$.

$$
\sum_{j=1}^{S} P_{i j} \bullet x_{i j} \geq R E C_{i}, \forall i \in T
$$

For information about the calculation of the received power $\mathrm{P}_{\mathrm{ij}}$, see Sections I and II.

The following constraint guarantees that the capacity bounds $\mathrm{W}_{\mathrm{j}}$ of the base stations are not violated.

$$
\sum_{i=1}^{T} w_{i j} \bullet x_{i j} \leq W_{j}, \forall j \in S
$$

Finally, we herein introduce a new maximization of communication capacity utilization constraint in the formalism as follows, based on equation (1a) above concepts.

$\sum^{S} \sum_{i=1}^{T}\left(w_{i j} \bullet x_{i j}-W_{j} \bullet Y_{j}\right)^{2}=0, \forall i \in T, j \in S$

However, if the solution of the problem is infeasible then an LP-relaxed version of it is solved. Nonetheless, only integer ( 0 or 1$)$ results are finally considered.

Considering the problem solution some (or all) base stations are pre-selected.

2) The saving in cost by dropping each pre-selected base station as well as the decrement of network communication capacity utilization, obtained by this dropping, are evaluated.

3) The base station whose removal saves the most units of cost and the least units of network communication capacity utilization is dropped.

4) A re-assignment of terminals to the remaining base stations takes place considering that the capacity bounds to the other base stations are not exceeded and that the reception power constraints are met (the power $\mathrm{P}_{\mathrm{ij}}$ emitted from the base station $\mathrm{j} \in \mathrm{S}$ and received by the terminal $\mathrm{i}$ $\in \mathrm{T}$ must be greater than or equal to the predefined 
reception threshold $\mathrm{REC}_{\mathrm{i}}$ ). For information regarding the calculation of the received power see Sections I and II.

5) After the drop of a base station, the savings by dropping the base station change and all potential savings are reevaluated by examining separately each of the terminals.

6) The algorithm proceeds until no more savings by dropping base stations can be obtained. Finally, all terminals will be connected to base stations, provided that there is enough capacity to the base stations, based on the above constraint

$$
\sum^{S} \sum_{i=1}^{T}\left(w_{i j} \bullet x_{i j}-W_{j} \bullet Y_{j}\right)^{2}=0, \forall i \in T, j \in S
$$

\section{Simulations \& Preliminary Test Results}

The objective of the tests was to evaluate the performance of the new heuristic algorithms QoS-HWNDA-1 and QoSHWNDA-2, compared to the older heuristics WNDA 1 and WNDA 2, proposed by the same authors in the past [14] as regards the design of minimum cost (cost-efficient) wireless access networks but ensuring maximization of network communication capacity utilization. For this purpose, the heuristics were compared with a slightly modified version of the optimization model described in Section II, in which the objective function was replaced by the following:

$$
\min Z=\left(\left(\sum_{j=1}^{S} C_{j} \bullet Y_{j}\right)+\left(\sum_{i=1}^{T} \sum_{j=1}^{S} c_{i j} \bullet x_{i j}\right)\right)
$$

Note also that for WNDA 2 / QoS-HWNDA-2, the LPrelaxed version of the TA ILP optimization problem solved in Step 1 of the algorithm (see Section IV) is used to the tests.

For the tests the software network design and simulation tool NetLab [15] was used. The experiments were run on an old PC equipped with a Pentium IV $3.2 \mathrm{GHz}$ processor and 512 MB RAM to show the feasibility even in older cheap hardware. Note that the solution of the optimization problem described in Section III and the heuristic algorithms is both CPU and memory intensive, especially when the number of the involved devices (base stations and terminals) is large.

The optimization problem, a LP-relaxed version of it and the two heuristics were applied in 50 randomly generated test topologies consisting of a number of wireless terminals and potential base station locations. All six methods had to select the best positions to place base stations and the best allocation of terminal(s) to them, so that the total base station placement cost to be minimum. The total number of base stations and wireless terminals in each topology varied from 10 to 400 . In order to validate the performance of the aforementioned methods, topology design costs were considered. Note that the design cost refers only to the total base station placement cost. Solution times for each method were also considered. Comparing the ILP optimization problem and its LP-relaxed version with the heuristic algorithms really provide an accurate validation of the performance of the developed heuristics.
A number of assumptions were made in order to support the network design process. In fact, the data rate produced by each terminal was $640 \mathrm{kbps}$ and the throughput capacity of each base station was $1024 \mathrm{Mbps}$.

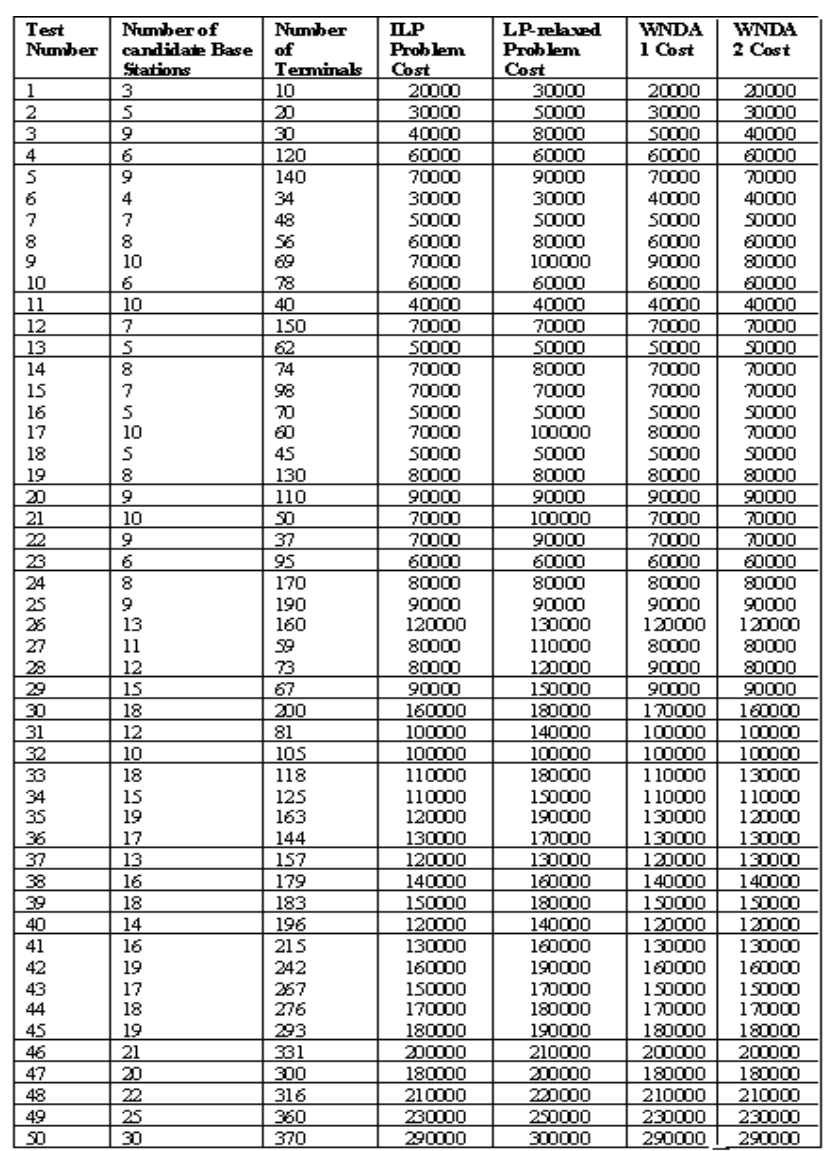

Table 1. Design costs for all basic test topologies and design methods

The cost of each base station was 10000 cost units. The diameter of each topology was in the range: $10-60 \mathrm{~km}$. It was assumed that each topology was placed in an open area. The network operates at the frequency of $2.4 \mathrm{GHz}$. The Ibrahim-Parsons propagation model for open areas (see [2] and Section III) was used for power reception calculation. The transmitted power by each base station was $10 \mathrm{dBm}$. The gain of each base station antenna was $40 \mathrm{dBi}$, while the gain of each terminal antenna was $30 \mathrm{dBi}$. Also, the actual height of the base station antennas was $25 \mathrm{~m}$, while the actual height of the terminal antennas was $0.01 \mathrm{~m}$. It was also: $\mathrm{REC}_{\mathrm{i}}=-50$ $\mathrm{dBm}$ for each terminal, land usage factor $\mathrm{L}=0.5 \%$ and height difference $\mathrm{H}=20 \mathrm{~m}$ (see Section II).

The test results are illustrated in Table 1, Table 2 and Figure 2. In Table 1 the design costs for each basic topology, but not the ones of the currently introduced QoS-HWNDA-1 and QoS-HWNDA-2, considering all the other basic aforementioned methods, can be seen; in Table 2 and Figure 2 the solution times for each topology, considering all the aforementioned methods, can be found. 
As it can be seen in the Table 1, the ILP optimisation problem presented the best results among all methods in all tests. On the other hand, the LP-relaxed version of the ILP optimisation problem presented the worst results. Actually, in the $66 \%$ of the total number of tests it presented worst results than the ILP optimisation problem. However, the older WNDA 2 heuristic algorithm as well as the newly one QoSHWNDA-2, presented results very close to these of the ILP optimisation problem. In fact, only in the $8 \%$ of the total number of tests, WNDA 2 presented worst results than these of the ILP optimisation problem. The same happens for the QoS-HWNDA-2 algorithm. The performance of the other heuristic algorithm namely, the WNDA 1 algorithm as well as that of the QoS-HWNDA-1 algorithm, was slightly worse than that this of the WNDA 2/ QoS-HWNDA-2 algorithms; in the $14 \%$ of the total number of tests, WNDA 1/ QoS-HWNDA-1 algorithms presented worst results than the ILP optimisation problem. Since WNDA 2 / QoS-HWNDA-2 are based on the solution of the LP-relaxed version of a TA ILP optimisation problem, whose formulation is similar to this of the standard base station placement and TA ILP optimisation problem, it is apparent that the algorithmic procedure involved in WNDA 2/ QoS-HWNDA-2 can substantially improve the design performance and the solutions provided by the LP-relaxed TA optimisation problem.

As it can be seen in the Table 2 and Figure 2, the solution times for the LP-relaxed version of the standard ILP optimization problem were smaller than these of all other methods in all tests. The solution times for the ILP optimization problem were small when the number of candidate base stations was small, but they grew almost exponentially when the number of candidate base stations was increased (see Figure 2). However, the solution times for WNDA 2/ QoS-HWNDA-2 were smaller than these for WNDA 1/ QoS-HWNDA-1. Actually, the solution times for the WNDA 2 / QoS-HWNDA-2 algorithms were similar to these for the ILP optimization problem in the cases where the number of candidate base stations was small, but they were substantially smaller than these for the ILP problem in the cases where the number of candidate base stations was above 12.

\begin{tabular}{|c|c|c|c|c|c|c|c|c|}
\hline \begin{tabular}{|l|}
$\begin{array}{l}\text { Number of } \\
\text { Test }\end{array}$ \\
\end{tabular} & \begin{tabular}{|l} 
Number of \\
candidate \\
Base \\
Stations \\
\end{tabular} & \begin{tabular}{|l|}
$\begin{array}{l}\text { Number of } \\
\text { Terminals }\end{array}$ \\
\end{tabular} & \begin{tabular}{|l|} 
ILP \\
Problem \\
(CPU time- \\
sec)
\end{tabular} & \begin{tabular}{|l|}
$\begin{array}{l}\text { LP-relaxed } \\
\text { Problem } \\
\text { (CPU time- } \\
\text { sec) }\end{array}$ \\
\end{tabular} & \begin{tabular}{|l|}
$\begin{array}{l}\text { WNDA 1 } \\
\text { (CPU time- } \\
\text { sec) }\end{array}$ \\
\end{tabular} & \begin{tabular}{|l|} 
WNDA 2 \\
(CPU time- \\
sec)
\end{tabular} & $\begin{array}{l}\text { QoS-HWNDA- } \\
1 \text { (CPU time- } \\
\text { sec) }\end{array}$ & $\begin{array}{l}\text { QoS-HWNDA } \\
2 \text { (CPU time- } \\
\text { sec) }\end{array}$ \\
\hline 1 & 3 & 10 & 1 & 1 & 1 & 1 & 1,5 & 1,5 \\
\hline 2 & 3 & 20 & 1 & 1 & 1 & 1 & 1,5 & 1,5 \\
\hline 3 & s & 30 & 1 & 1 & 1 & 1 & 1,5 & 1,5 \\
\hline 4 & 7 & 120 & 2 & 1 & 4 & 2 & 4,5 & 2,5 \\
\hline 5 & 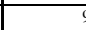 & 140 & 3 & 1 & 6 & 3 & 6,5 & 3,5 \\
\hline 6 & 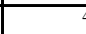 & 34 & 1 & 1 & 1 & 1 & 1,5 & 1,5 \\
\hline 7 & 7 & 48 & 1 & 1 & 2 & 1 & 2,5 & 1,5 \\
\hline 8 & 8 & 56 & 1 & 1 & 2 & 1 & 2,5 & 1,5 \\
\hline 9 & 10 & 69 & 2 & 1 & 4 & 2 & 4,5 & 2,5 \\
\hline 10 & 5 & 78 & 1 & 1 & 2 & 1 & 2,5 & 1,5 \\
\hline 11 & 16 & 40 & 1 & 1 & 1 & 1 & 1,5 & 1,5 \\
\hline 12 & 7 & 150 & 2 & 1 & 5 & 2 & 5,5 & 2,5 \\
\hline 13 & 5 & 62 & 1 & 1 & 2 & 1 & 2,5 & 1,5 \\
\hline 14 & 8 & 74 & 1 & 1 & 2 & 1 & 2,5 & 1,5 \\
\hline 15 & ? & 98 & 2 & 1 & 3 & 2 & 3,5 & 2,5 \\
\hline 16 & 5 & 70 & 1 & 1 & 2 & 1 & 2,5 & 1,5 \\
\hline 17 & 10 & 60 & 2 & 1 & 3 & 2 & 3,5 & 2,5 \\
\hline 18 & 3 & 45 & 1 & 1 & 1 & 1 & 1,5 & 1,5 \\
\hline 19 & 8 & 130 & 2 & 1 & 5 & 2 & 5,5 & 2,5 \\
\hline 20 & 9 & 110 & 2 & 1 & 5 & 2 & 5,5 & 2,5 \\
\hline 21 & 10 & 50 & 1 & 1 & 2 & 1 & 2,5 & 1,5 \\
\hline 22 & 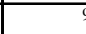 & 37 & 1 & 1 & 2 & 1 & 2,5 & 1,5 \\
\hline 23 & 7 & 95 & 1 & 1 & 3 & 1 & 3,5 & 1,5 \\
\hline 24 & 8 & 170 & 3 & 1 & 7 & 3 & 7,5 & 3,5 \\
\hline 25 & 9 & 190 & 4 & 2 & 9 & 4 & 10 & 5 \\
\hline 26 & 13 & 160 & 10 & 2 & 12 & 4 & 13 & 5 \\
\hline 27 & 11 & 59 & 2 & 1 & 3 & 2 & 3,5 & 2,5 \\
\hline 28 & 12 & 73 & 3 & 1 & 5 & 2 & 5,5 & 2,5 \\
\hline 29 & 15 & 67 & 10 & 1 & 5 & 3 & 5,5 & 3,5 \\
\hline 30 & 18 & 200 & 171 & 3 & 24 & 6 & 25,5 & 7,5 \\
\hline 31 & 12 & 81 & 7 & 1 & 6 & 3 & 6,5 & 3,5 \\
\hline 32 & 10 & 105 & 3 & 1 & 6 & 2 & 6,5 & 2,5 \\
\hline 33 & 18 & 118 & 80 & 2 & 12 & 4 & 13 & 5 \\
\hline 34 & 15 & 125 & 15 & 2 & 9 & 4 & 10 & 5 \\
\hline 35 & 15 & 163 & 175 & 2 & 17 & 6 & 18 & 7 \\
\hline 36 & 17 & 144 & 118 & 2 & 15 & 4 & 16 & 5 \\
\hline 37 & 13 & 157 & 14 & 2 & 12 & 3 & 13 & 4 \\
\hline 38 & 16 & 179 & 67 & 2 & 19 & 4 & 20 & 5 \\
\hline 39 & $\overline{18}$ & 183 & 130 & 2 & 24 & 5 & 25 & 6 \\
\hline 40 & 14 & 196 & 17 & 2 & 16 & 5 & 17 & 6 \\
\hline 41 & 16 & 215 & 43 & 3 & 21 & 6 & 22,5 & 7,5 \\
\hline 42 & $\overline{15}$ & $\overline{242}$ & 178 & 3 & 34 & 7 & 35,5 & 8,5 \\
\hline 43 & 17 & 267 & 92 & 3 & 33 & 7 & 34,5 & 8,5 \\
\hline 44 & 18 & 276 & 211 & 4 & 42 & 8 & 44 & 10 \\
\hline \begin{tabular}{l|}
45 \\
\end{tabular} & $\frac{15}{15}$ & 293 & 294 & 4 & 43 & 8 & 45 & 10 \\
\hline 46 & 21 & 331 & 624 & 5 & 55 & 10 & 57,5 & 12,5 \\
\hline 47 & 26 & 300 & 233 & 4 & 54 & 10 & 56 & 12 \\
\hline 48 & 22 & 316 & 548 & 5 & 59 & 10 & 61,5 & 12,5 \\
\hline \begin{tabular}{|l|}
49 \\
\end{tabular} & 25 & 360 & $\begin{array}{ll}1598 \\
\end{array}$ & 6 & 82 & 14 & 85 & 17 \\
\hline 50 & 30 & 370 & 7224 & 7 & 93 & 17 & 96,5 & 20,5 \\
\hline
\end{tabular}

Table 2. Solution times for all test topologies and design methods 


\section{SOLUTION TIMES EVOLUTION WITH RESPECT TO THE NUMBER OF TERMINALS AND BASE STATIONS OF THE WIRELESS NETWORK}

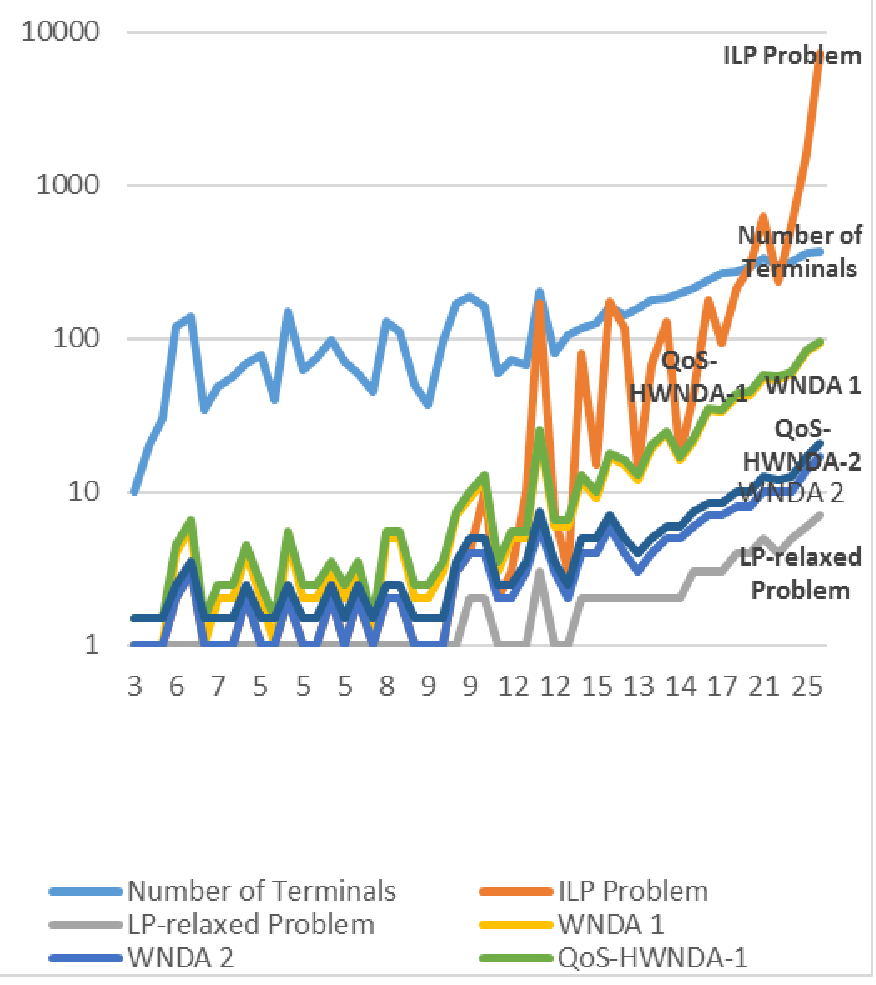

Figure 2. Graphical representation of the results in Table 2

\section{CONCLUSIONS AND FUTURE PROSPECTS}

In this paper two novel heuristic algorithms for the design of wireless access network are presented, namely the QoSHWNDA-1 (improved QoS variant of the older WNDA 1) and QoS-HWNDA-2 (improved QoS variant of the older WNDA 2). Both new algorithms are based on Graph Theory [13] and they are greedy as their older counterparts. In their formulation, except from capacity constraints, wireless reception constraints are also included as well as the newly herein introduced constraint of maximization of network communication capacity utilization plays an important role in supporting QoS functionality in the new proposed formalism.

Important experimental results were obtained for the developed wireless access network design methods as it can be seen in Section V. The developed heuristic algorithms were compared with the standard base station placement and TA ILP optimization problem (see Section II) and its LP-relaxed version through a series of tests.

From the tests it is understood that the wireless access planning heuristics are capable of solving medium $(\sim 50$ network devices) or even large (> 100 devices) network design problems in polynomial time, providing results very close to these of the standard ILP optimization problem. However, WNDA 2/ QoS-HWNDA-2 outperformed WNDA 1 / QoS-HWNDA-1 regarding design cost. Specifically, only in a small number of tests (8-9 \%), WNDA 2 / QoS-HWNDA-2 presented worst results than these of the ILP optimization problem. The performance of the WNDA 1 / QoS-HWNDA-1 algorithm was slightly worse that this of the WNDA 2 / QoS-HWNDA-2 algorithm; in the 14-15\% of the tests, WNDA 1 / QoSHWNDA-1 presented worst results than the ILP optimization problem.

The solution times for the heuristics were small. Actually, the solution times for the WNDA 2 / QoS-HWNDA-2 algorithm were similar to these for the ILP optimization problem when the number of candidate base stations in the tests was small, but they were substantially smaller than these for the ILP optimization problem when the number of candidate base stations was quite larger (above 12). However, the solution times for the WNDA 1 / QoSHWNDA-1 algorithm were larger than these for the WNDA 2 / QoS-HWNDA-2 algorithm in most cases.

The algorithmic procedure involved in WNDA 2 / QoSHWNDA-2 can substantially improve the design performance and the solutions provided by the LP-relaxed TA optimization problem solved in Step 1 of WNDA 2 / QoS-HWNDA-2 (see Section IV). This problem is similar to the LP-relaxed version of the ILP optimization problem used to the tests, which alone presented the worst results among all design methods.

Note that complex propagation phenomena, such as radio interference, were not taken into account to the developed wireless access network design methods. These phenomena cannot be explicitly included in linear constraints or mathematical expressions since they can only be adequately represented by complex non-linear formulations.

However, the research concerning the improvement of the two developed methods continues. New constraints that consider phenomena like interference are studied and will be presented in the near future. Besides, the developed design methods can be customized and properly modified so that to be used with specific wireless network technologies. For example, a special version of WNDA 2 / QoS-HWNDA-2 can be used for designing indoor CDMAbased networks, another for fixed wireless network planning etc. But the most important work to be done is to work with huge wireless networks applying the above formalisms efficiently. 


\section{REFERENCES}

[1] Xichun Li; Abudulla Gani; Rosli Salleh; Omar Zakaria (February 2009). The Future of Mobile Wireless Communication Networks (PDF). International Conference on Communication Software and Networks. ISBN 978-0-7695-3522-7. Retrieved 27 September 2013.

[2] John Proakis, Masoud Salehi. " Digital Communications//;, 5th Edition (Irwin Electronics \& Computer Enginering) 5th Edition, 2007

[3] Abdessadeq Fettouh; Abdelaziz El Faziki; Najib El Kamoun, "Improving horizontal handover performance of real time applications over homogeneous wireless networks", SETIT-2012, IEEEXplore, Publication Year: 2012, Page(s):619 - 623, 21-24/3/2012

[4] Oumarou Halidou ; Radouane Mrabet, "End to end IP over IEEE 802.16 QoS framework", SETIT-2012, IEEEXplore, Publication Year: 2012, Page(s):685 - 689

[5] M. A. M. M. El-Bendary; A. Haggag; H. B. Kazemian, "Activate the CQDDR role for improving throughput over IEEE 802.15.1 wireless links", SETIT-2012, IEEEXplore, Publication Year: 2012, Page(s):663 $-668$

[6] J.K.L. Wong, M.J. Neve and K.W. Sowerby, "Optimisation strategy for wireless communications system planning using linear programming", Electronic Letters, Vol. 37, No.17, pp. 1086-1087, $16^{\text {th }}$ August 2001.

[7] E. Amaldi, A. Capone, and F. Malucelli, "Planning UMTS base station location: Optimisation models with power control and algorithms", IEEE Transactions on Wireless Communications, Vol. 2, No. 5, pp. 939952, September 2003.

[8] Haesik Kim, "Wireless Communications Systems Design and Considerations", Published Online: 7 AUG 2015,
DOI: 10.1002/9781118759479.ch12, Copyright (C) 2015 John Wiley \& Sons, Ltd.

[9] Egli, "Radio Propagation Above 40 MC Over Irregular Terrain" Proceedings IRE, Vol. 45, pp. 1383-1391, October 1957.

[10] CCIR, XVII ${ }^{\text {th }}$ Plenary Assembly, Duselldorf, 1990, Vol. V, Rep. 5673, Rec. 3704, Rep. 2394.

[11] M. Hata, "Empirical Formula for Propagation Loss in Land Mobile Radio Services", IEEE Transactions in Vehicular Technology, Vol. 29, No. 3, 1980.

[12] COST 231 Final Report, "Digital mobile radio: COST 231 view on the evolution towards 3rd generation systems". Editors: Damosso, E. and Correia L.M., European Commission - COST Telecommunications, Brussels, Belgium, 1998.

[13] Aaron Kershenbaum, "Telecommunications Network Design Algorithms", McGraw-Hill International Editions, 1993.

[14] Vasilios Pasias ; Dimitrios A. Karras ; R.C. Papademetriou, "Heuristic Algorithms for Efficient Wireless Multimedia Network Design", Software Engineering and Advanced Applications, 2006. SEAA '06. 32nd EUROMICRO Conference, Date of Conference: 29 Aug.-1 Sept. 2006, Date Added to IEEE Xplore: 16 October 2006, INSPEC Accession Number: 9165264, DOI: 10.1109/EUROMICRO.2006.40, Publisher: IEEE, Conference Location: Cavtat, Dubrovnik, Croatia

[15] R.C. Papademetriou and V. Pasias, "NetLab - An Interactive Simulation Environment for Communication Networks", Proceedings of the 1st IEEE International Conference on Information Technology: Research and Education (ITRE 2003), NJ, USA, August 11 - 13, 2003, pp. 123 127. 